\title{
Little Rip and Pseudo Rip phenomena from coupled dark energy
}

\author{
I. Brevik! \\ Department of Energy and Process Engineering, Norwegian University of \\ Science and Technology, N-7491 Trondheim, Norway \\ A. V. Timoshkin and Y. Rabochaya 2 \\ Tomsk State Pedagogical University, 634061 Tomsk, Russia
}

\begin{abstract}
We consider Little Rip (LR) and Pseudo Rip (PR) cosmological models with two interacting ideal fluids, corresponding to dark energy and dark matter. The interaction between the dark energy and the dark matter fluid components is described in terms of the parameters in the equations of state for the LR and PR universes. In contrast to a model containing only a pure dark energy, the presence of the interaction term between the fluid components in the gravitational equations leads to a modification of the equation of state parameters. The properties of the early universe in this formalism are pointed out.
\end{abstract}

\section{Introduction}

In the present paper we study a cosmological system containing two coupled fluids: a dark energy component with a linear inhomogeneous equation of state (EoS) and a dark matter component with a linear homogeneous EoS. As is known, a dark energy universe shows a peculiar behavior with respect to singularities in the far future, even in cases where it is induced by modified gravity [1] (for reviews, see [2, 3, 4, 5, 6]). Recently, a general review of dark energy was given in Ref. [7]. It is of interest to investigate the influence of

\footnotetext{
${ }^{1}$ iver.h.brevik@ntnu.no

${ }^{2}$ Also at Department of Physics, University of Trento, Trento, Italy.
} 
a coupling between dark energy and dark matter responsible for the accelerated expansion of the universe. Among various different possible models investigated in the literature, we shall focus attention on those that treat dark energy and dark matter as ideal (nonviscous) fluids with an unusual EoS. Very general dark fluid models with an inhomogeneous EoS were introduced in [8, 9, 10, 11]. There exist various cosmological scenarios for the evolution of the universe including the Big Rip [12, 13, 14, the Little Rip [15, 16, 17, 18, 19, 20, 21, 22, the Pseudo Rip 23] and the Quasi Rip [24.

In the present work we investigate the influence from the interaction between dark energy and dark matter via the time-dependent thermodynamic parameter $w$ and the cosmological constant $\Lambda$. That means, the EoS will play an important role, for the LR as well as for the PR phenomena. For the two fluid components we obtain expressions for the dark energy density, the dark matter density, and the cosmological 'constant', called $\Lambda(t)$. In particular, we derive the parameters for the early stage of the history of the universe.

\section{Little Rip models with interaction between dark energy and dark matter}

We consider a universe filled with two interacting ideal fluids components: a dark energy, and a dark matter, in a spatially flat Friedman-RobertsonWalker metric with scale factor $a$. The background equations are given by [1]

$$
\begin{aligned}
& \dot{\rho}+3 H(\rho+p)=-Q, \\
& \dot{\rho}_{m}+3 H\left(\rho_{m}+p_{m}\right)=Q, \\
& \dot{H}=-\frac{1}{2} k^{2}\left(\rho+p+\rho_{m}+p_{m}\right),
\end{aligned}
$$

where $H=\dot{a} / a$ is the Hubble rate and $k^{2}=8 \pi G$ with $G$ being Newton's gravitational constant. Moreover $\rho, p$ and $\rho_{m}, p_{m}$ are the energy density and the pressure of a dark energy and a dark matter, respectively, and $Q$ is the interaction term between dark energy and dark matter. A dot denotes the derivative with respect to cosmic time $t$.

Friedman's equation for the Hubble rate is

$$
H^{2}=\frac{1}{3} k^{2}\left(\rho+\rho_{m}\right) .
$$


We will now investigate various cosmological models.

\subsection{Little Rip model: first variant}

Let us consider a LR model with the following form for Hubble parameter [17]:

$$
H=H_{0} \exp (\lambda t), \quad H_{0}>0, \lambda>0 .
$$

We will follow the development of the universe beginning at some time $t=0$ which will at first be left unspecified, except that it refers to an initial instant in the very early universe. Its precise meaning will be dependent on which model we consider. The symbol $H_{0}$ means the Hubble parameter at this particular instant.

Now assume that the dark energy obeys an inhomogeneous EoS [8]:

$$
p=w(t) \rho+\Lambda(t)
$$

and that the dark matter obeys another inhomogeneous EoS [1]:

$$
p_{m}=\tilde{w}(t) \rho_{m} .
$$

The gravitational equation of motion for dark matter can then be written as

$$
\dot{\rho}_{m}+3 H(1+\tilde{w}) \rho_{m}=Q
$$

We will assume that the thermodynamic parameter $\tilde{w}(t)$ for dark matter has the following form:

$$
\tilde{w}(t)=-1+\exp (-\lambda t) .
$$

It means that $\tilde{w}$ is equal to zero at the initial instant $t=0$, and is thereafter decreasing asymptotically to -1 in the far future.

As for the interaction term between dark energy, we will take it to depend linearly on time:

$$
Q=Q_{1} t+Q_{2},
$$

where $Q_{1}$ and $Q_{2}$ are constants. A motivation for this choice can be found in modified gravity theory [2, 5].

The solution of equation (6) can be written as

$$
\rho_{m}(t)=\frac{1}{3 H_{0}}\left[\tilde{Q}_{2}\left(1-\exp \left(-3 H_{0} t\right)\right)+Q_{1} t\right]
$$


where $\tilde{Q}_{2} \equiv Q_{2}-Q_{1} /\left(3 H_{0}\right)$. If $t \rightarrow 0$, then $\rho_{m} \rightarrow 0$. We thus see what is the physical meaning of the instant $t=0$ in this model: it is the time at which dark matter starts to appear in the universe. When $t \rightarrow \infty, \rho_{m} \rightarrow\left(Q_{1} / 3 H_{0}\right) t$.

Taking into account equations (2)-(4) we obtain the gravitational equation of motion for the dark energy,

$$
\frac{6 \lambda H^{2}}{k^{2}}-\dot{\rho}_{m}+3 H\left[(1+w)\left(\frac{3}{k^{2}} H^{2}-\rho_{m}\right)+\Lambda\right]=-Q .
$$

Let us choose the parameter $w(t)$ for dark energy in the form

$$
w(t)=-1-\frac{1}{3 k^{2} H^{2}} .
$$

It means that the deviation from the phantom divide $w=-1$ of the dark energy fluid is at maximum at the initial instant $t=0$, and approaches the limit -1 asymptotically in the far future. From equation (10) we obtain

$$
\Lambda(t)=\frac{1}{k^{2}}\left(\frac{1}{k^{2}}-2 \lambda H\right)-\frac{H_{0}}{H}\left(1+\frac{1}{3 k^{2} H H_{0}}\right) \rho_{m} .
$$

In particular, at $t=0$ where $w(0) \equiv w_{\text {in }}$ and $\Lambda(0) \equiv \Lambda_{\text {in }}$ we have

$$
w_{\text {in }}=-1-\frac{1}{3 k^{2} H_{0}^{2}}, \quad \Lambda_{\text {in }}=\frac{1}{k^{2}}\left(\frac{1}{k^{2}}-2 \lambda H_{0}\right) .
$$

When $t \rightarrow \infty$, one sees that $\Lambda(t) \rightarrow-\infty$.

\subsection{Little Rip model: second variant}

Next we will investigate another example of a LR model in which the parameter $H$ equals [17]

$$
H(t)=H_{0} \exp (C \exp (\lambda t)),
$$

where $H_{0}, C$ and $\lambda$ are positive constants. We start from $t=0$, as above. The increase of $H(t)$ with time assumed in equation (14) is seen to be much stronger than it was in equation (3).

For the thermodynamic dark matter parameter $\tilde{w}(t)$ in the EoS (15) we assume now the form

$$
\tilde{w}(t)=-1+\exp (\lambda t)
$$


Note that $\tilde{w}(t)$ is always zero or positive: it is zero at the initial instant $t=0$; thereafter it increases exponentially with increasing $t$.

As for the interaction term $Q$ we take it to be proportional to the Hubble parameter $H$ times an exponential of $\lambda t$ :

$$
Q=\frac{Q_{0} H}{H_{0}} \exp (\lambda t)
$$

$Q_{0}$ being an unspecified constant.

Solving the gravitational equation of motion (6) for dark matter, we find

$$
\rho_{m}(t)=\frac{Q_{0}}{3 H_{0}}\left\{1-\exp \left(\frac{3 H_{0}}{C \lambda} \exp (C[1-\exp (C[\exp (\lambda t)-1])])\right)\right\} .
$$

We take the parameter $w(t)$ for dark energy in equation (4) to have the same form (11) as before. Taking into account equations (11), (16) and (17), we then obtain from equation (10)

$$
\Lambda(t)=\frac{1}{k^{2}}\left[\frac{1}{k^{2}}-2 C \lambda H \exp (\lambda t)\right]-\left[\exp (\lambda t)+\frac{1}{3 k^{2} H^{2}}\right] \rho_{m} .
$$

In the early universe at $t=0$ we get

$$
w_{\text {in }}=-1-\frac{1}{3 k^{2} H_{0}^{2} \exp (2 C)}, \quad \Lambda_{\text {in }}=\frac{1}{k^{2}}\left[\frac{1}{k^{2}}-2 C \lambda H_{0} \exp (C)\right] .
$$

These expressions are to be compared with expressions (13) above.

In contrast to the previous model, there is no special property possessed by $\rho_{m}$ at the instant $t=0$ in this model. When $t=0$ equation (17) simply yields

$$
\rho_{m}(0)=\frac{Q_{0}}{3 H_{0}}\left\{1-\exp \left(\frac{3 H_{0}}{C \lambda}\right)\right\} .
$$

In the far future, $t \rightarrow \infty$, we see moreover that the dark matter gradually fades away, $\rho_{m}(\infty)=0$. This can be looked upon as a characteristic property analogous to the property $\rho_{m}(0)=0$ in our previous model. It should be noted that for both models, $p_{m}(0)=0$.

When $t \rightarrow \infty$, we again find that $\Lambda(t) \rightarrow-\infty$.

So far we have thus constructed two examples of LR cosmology, in terms of time-dependent parameters in the EoS, taking into account an interaction term $Q$ between dark energy and dark matter. 


\section{Pseudo Rip model}

We will now investigate examples in which the Hubble parameter approaches a constant in the far future. That means, the universe approaches asymptotically a de Sitter space. We will make this analysis in analogy with the LR model above.

First example. Assume that the Hubble parameter is given as [17]

$$
H=H_{0}-H_{1} \exp (-\lambda t)
$$

where $H_{0}, H_{1}$ and $\lambda$ are positive constants, $H_{0}>H_{1}$, and $t>0$.

Let us suppose that the parameter $\tilde{w}(t)$ for dark matter in (5) has the form (15). Choosing the interaction between dark energy and dark matter in the form

$$
Q=\frac{3 Q_{0}}{H_{0}} H \exp (\lambda t)
$$

we find that the solution of the gravitational equation (6]) is given by

$$
\rho_{m}(t)=\frac{Q_{0}}{H_{0}}\left\{1-\exp \left[-3\left(\frac{H_{0}}{\lambda}(\exp (\lambda t)-1)\right)-H_{1} t\right]\right\} .
$$

According to this expression the dark matter energy density is thus $\rho_{m}=0$ at $t=0$ and $\rho_{m}=1$ at $t=\infty$.

If the thermodynamic parameter $w(t)$ for dark energy has the form (11), we obtain from (10) the following expression for the cosmological 'constant':

$$
\Lambda(t)=\frac{1}{k^{2}}\left[\frac{1}{k^{2}}-2 \lambda H_{1} \exp (-\lambda t)\right]+(w-\tilde{w}) \rho_{m} .
$$

In the early universe $t=0$ we have

$$
w_{\text {in }}=-1-\frac{1}{3 k^{2}\left(H_{0}-H_{1}\right)^{2}}, \quad \Lambda_{\text {in }}=\frac{1}{k^{2}}\left(\frac{1}{k^{2}}-2 \lambda H_{1}\right) .
$$

Second example. This example considers a cosmological model with asymptotically de Sitter evolution [20]. The Hubble parameter is taken equal to 25

$$
H=\frac{x_{f}}{\sqrt{3}}\left[1-\left(1-\frac{x_{0}}{x_{f}}\right) \exp \left(-\frac{\sqrt{3} A t}{2 x_{f}}\right)\right]
$$


where $x_{0}=\sqrt{\rho_{0}}$ is the energy density at present, $x_{f}$ is a finite value related to divergence of the cosmological time [25], and $A$ is a positive constant. When $t \rightarrow \infty$ the Hubble parameter $H \rightarrow x_{f} / \sqrt{3}$ and the expression (26) asymptotically tends to the de Sitter solution.

Let us take the parameter $\tilde{w}(t)$ in the form

$$
\tilde{w}(t)=-1+\exp \left(\frac{\sqrt{3} A t}{2 x_{f}}\right)
$$

and take the interaction term in the form

$$
Q=Q_{0} \exp \left(-\frac{2 x_{f}^{2}}{A} \exp \left(\frac{\sqrt{3} A t}{2 x_{f}}\right)\right)
$$

where $Q_{0}$ is a constant.

In this case the solution of the gravitational equation (6) for dark matter becomes

$$
\rho_{m}(t)=\frac{Q}{\sqrt{3}\left(x_{f}-x_{0}\right)}\left[\exp \left(\sqrt{3}\left(x_{f}-x_{0}\right) t\right)-1\right] .
$$

using (6) , (11) and (29) to solve (10) with respect to $\Lambda(t)$, we obtain

$$
\Lambda(t)=\frac{1}{k^{2}}\left[\frac{1}{k^{2}}-A\left(1-\frac{\sqrt{3}}{x_{f}} H\right)\right]-\left[\frac{1}{3 k^{2} H^{2}}+\frac{x_{f}-x_{0}}{x_{f}-\sqrt{3} H}\right] \rho_{m} .
$$

In the early universe one gets

$$
w_{\text {in }}=-1-\frac{1}{3 k^{2} x_{0}^{2}}, \quad \Lambda_{\text {in }}=\frac{1}{k^{2}}\left[\frac{1}{k^{2}}-A\left(1-\frac{x_{0}}{x_{f}}\right)\right] .
$$

Thus we have investigated, via the parameters of the EoS, the influence of the dark energy/dark matter coupling on the evolution of the Pseudo Rip model.

\section{Conclusion}

In the present work we studied Little Rip and Pseudo Rip cosmological models taking into account the coupling between dark energy and dark matter. The gravitational equations of motion for dark matter were solved, and the 
descriptions of the LR and PR universes in terms of the EoS parameters were given. Unlike a model containing only a pure dark energy, the presence of an interaction term between dark energy and dark matter in the gravitational equations leads to corrections in the EoS parameters; see Refs. [25, 26, 27, 28]. The case of the very early universe was emphasized.

We mention finally that there exist also other investigations dealing with the coupling between the dark energy and dark matter components, from various perspectives; cf., for instance, the Archimedean-type approach followed by Balakin and Bochkarev [29, 30].

\section{Acknowledgement}

This work has been supported by project 2.1839.2011 of Ministry of Education and Science (Russia) and LRSS project 224.2012.2 (Russia). We thank Professor Sergei Odintsov for very useful discussions and valuable remarks.

\section{References}

[1] Nojiri S., Odintsov S.D.,Tsujikawa S. Properties of singularities in (phantom) dark energy uni-verse. Phys.Rev. D 71, 063004, (2005), arXiv: 0501025 [hep-th].

[2] Nojiri S., Odintsov S.D. Introduction to modified gravity and gravitational alternative for dark energy. Int. J. Geom. Meth. Mod. Phys. 4, 115-146, (2007).

[3] Capozziello M, De Laurentis M, Faraoni, V. A bird's eye view of $f(R)$ gravity. arXiv:0909.4672 [gr-qc].

[4] Capozziello M, Faraoni V. Beyond Einstein gravity: A survey of gravitational theories for cosmology and astrophysics. Inspirehep.net/record/1107700.

[5] Nojiri S., Odintsov S.D. Unified cosmic history in modified gravity: from F(R) theory to Lorentz non-invariant models. Phys. Rept. 505, 59-144, (2011), arXiv:1011.0544 [gr-qc].

[6] Nojiri S., Odintsov S. D. Accelerating cosmology in modified gravity: from convenient $F(R)$ or string inspired theory to bimetric $F(R)$ gravity, arXiv:1306.4426 [gr-qc]. 
[7] Bamba K., Capozziello S., Nojiri S., Odintsov S. D. Dark energy cosmology: The equivalent description via different theoretical models and cosmography tests. Astrophys. Space Sci. 342, 155-228 (2012), arXiv:1205.3421 [gr-qc].

[8] Nojiri S., Odintsov S.D. Inhomogeneous equation of state of the Universe: Phantom era, future singularity and crossing the phantom b arrier. Phys. Rev. D 72, 023003, (2005), arXiv:0505215 [hep-th].

[9] Nojiri S., Odintsov S.D. The new form of the equation of state for dark energy fluid and accelerating universe. Phys. Lett. B 639, 144-150, (2006).

[10] Brevik I., Nojiri S., Odintsov S.D., Vanzo L. Entropy and universality of Cardy-Verlinde formula in dark energy universe. Phys. Rev. D 70, 043520, (2004), arXiv: 0401073 [hep-th].

[11] Capozziello S., Cardone V., Elizalde E., Nojiri S, Odintsov S.D. Observational constraints on dark energy with generalized equation of state. Phys. Rev. D 73, 043512, (2006), arXiv:0508350[hep-th].

[12] Caldwell R.R., Kamionkowski M., Weinberg N.N. Phantom Energy and Cosmic Doomsday. Phys. Rev. Lett. 91, 071301, 2003 astro-ph/0302505.

[13] Nojiri S., Odintsov S.D. Final state and thermodynamics of a dark energy universe, Phys. Rev. D 70, 103522, 2004.

[14] Nojiri, S., Odintsov, S. D. Quantum de Sitter cosmology and phantom matter, Phys. Lett. B 562, 147-152, (2003).

[15] Frampton H., Ludwick K.J., Scherrer R.J. The Little Rip. Phys. Rev. D 84, 063003, (2011), arXiv:1106.4996v1 [astro-ph. CO].

[16] Brevik I., Elizalde E., Nojiri S. and Odintsov S. D. Viscous Little Rip cosmology. Phys. Rev. D 84, 103508 (2011), arXiv:1107.4642 [hep-th].

[17] Frampton P.H., Ludwick K.J., Nojiri S., Odintsov S.D., Scherrer R.J. Models for Little Rip dark energy. Phys. Lett. B 708, 204-211 (2012), arXiv:1108.0067v2 [hep-th]. 
[18] Astashenok A.V., Nojiri S., Odintsov S.D., Yurov A.V. Phantom cosmology without Big Rip singularity. Phys. Lett. B 709, 396-403 (2012), arXiv:1201.4056v2 [gr-qc].

[19] Astashenok A.V., Nojiri S., Odintsov S.D., Scherrer R.J. Scalar dark energy models mimicking $\Lambda$ CDM with arbitrary future evolution. arXiv:1203.1976v2 [gr-qc]. To appear in Phys. Lett. B.

[20] Astashenok A.V., Elizalde E., Odintsov S.D., Yurov A.V. Equation-ofstate formalism for dark energy models on the brane and the future of brane universes. Eur. Phys. J. C 72, 2260 (2012), arXiv:1206.2192v1 [gr-qc].

[21] Nojiri S., Odintsov S.D., Saez-Gomez D. Cyclic, ekpyrotic and little rip universe in modified gravity. arXiv:1108.0767v2 [hep-th].

[22] Makarenko A.N., Obukhov V.V., Kirnos I. V. From Big to Little Rip in modified F(R,G) gravity. arXiv:1201.4742v2 [gr-qc].

[23] Frampton P.H., Ludwick K.J., Scherrer R.J. Pseudo-rip: Cosmological models intermediate between the cosmological constant and the little rip. Phys. Rev. D 85, 083001, (2012), arXiv:1112.2964v2 [astro-ph. CO].

[24] Wei H., Wang L.F., Guo X.J. Quasi-Rip: a new type of rip model without cosmic dooms-day. Phys. Rev. D 86, 083003 (2012), arXiv:1207.2898,v1 [gr-qc].

[25] Brevik I., Obukhov V.V., Timoshkin A.V. Quasi-Rip and Pseudo-Rip universes induced by the fluid inhomogeneous equation of state. Astrophys. Space Sci. 344, 275-279 (2013).

[26] Brevik I., Obukhov V.V., Osetrin K.E., Timoshkin, A.V. Little Rip cosmological models with time-dependent equation of state. Mod. Phys.Lett. A 27, 1250210 (2012).

[27] Brevik I., Obukhov V.V., Timoshkin A.V. Rabochaya Y. Rip brane cosmology from 4d inhomogeneous dark fluid universe. Astrophys. Space Sci. 346, 267-271 (2013).

[28] Brevik I., Timoshkin A.V., Rabochaya Y., Zerbini S. Turbulence accelerating cosmology from an inhomogeneous dark fluid. Astrophys. Space Sci. 347, 203-208 (2013). 
[29] Balakin A. B., Bochkarev V. V. Archimedean-type force in a cosmic dark fluid. I. Exact solutions for the late-time accelerated expansion. Phys. Rev. D 83, 024035 (2011).

[30] Balakin A. B., Bochkarev V. V. Archimedean-type force in a cosmic dark fluid. II. Qualitative and numerical study of a multistage universe expansion. Phys. Rev. D 83, 024036 (2011). 